\title{
The archetypal equation and its solutions attaining the global extremum
}

\author{
MARIUsz SudzIK(D)
}

\begin{abstract}
Let $(\Omega, \mathcal{F}, \mathbb{P})$ be a probability space and let $\alpha, \beta: \mathcal{F} \rightarrow \mathbb{R}$ be random variables. We provide sufficient conditions under which every bounded continuous solution $\varphi: \mathbb{R} \rightarrow \mathbb{R}$ of the equation $\varphi(x)=\int_{\Omega} \varphi(\alpha(\omega)(x-\beta(\omega))) \mathbb{P}(d \omega)$ is constant. We also show that any non-constant bounded continuous solution of the above equation has to be oscillating at infinity.
\end{abstract}

Mathematics Subject Classification. Primary 39B22, Secondary 39B05.

Keywords. Archetypal equation, Linear functional equation, Equation with rescaling, Iterative equation, Bounded continuous function, Global extremum.

\section{Introduction}

The paper concerns the linear functional equation of infinite order

$$
\varphi(x)=\iint_{\mathbb{R}^{2}} \varphi(a(x-b)) \mu(d a, d b),
$$

where $\mu$ is a given Borel probability measure on $\mathbb{R}^{2}$. We can think about equation (1.1) also in the language of random variables. Given a probability space $(\Omega, \mathcal{F}, \mathbb{P})$ let $(\alpha, \beta): \Omega \rightarrow \mathbb{R}^{2}$ be a fixed random vector with a distribution $\mu$. Then the equation

$$
\varphi(x)=\int_{\Omega} \varphi(\alpha(\omega)(x-\beta(\omega))) \mathbb{P}(d \omega)
$$

is equivalent to (1.1).

The study of equation (1.1) was initiated by Derfel [5] in 1989. He considered equation (1.2) under the additional assumption $\alpha>0$ a.s. and he noticed 
that the behaviour of solutions of (1.1) crucially depends on the value of the integral

$$
K:=\iint_{\mathbb{R}^{2}} \ln |a| \mu(d a, d b) .
$$

He proved that, under some additional technical assumptions, if $K \in(-\infty, 0)$ then equation (1.1) has only trivial, i.e. constant, solutions in the class of bounded continuous functions. If $K \in(0, \infty)$ and $\alpha>0$ a.s., then he constructed a non-trivial bounded continuous solution. The details of this theorem and its proof are included in [4], Theorem 1.1.

Many deep results connected with equation (1.1) were obtained and collected by Bogachev et al. [3] and [4]. In those papers equation (1.1) was named by the authors as the archetypal equation since it is a rich source of many famous functional and differential-functional equations. For instance, one can obtain a balanced version of the pantograph equation; details can be found in [4]. It is worth noting that functional equations of a more general form than (1.2) were also considered in the literature; an interested reader, can find them e.g. among papers written by Polish mathematicians from the Silesian University: Baron, Kapica and Morawiec (see, for instance, [2] and [8]). Moreover, a lot of interesting remarks connected with the archetypal equation, its special cases and equations of similar forms can be found in [1] and [6].

Here we can confine ourselves to (1.1) since equations with linearly transformed arguments are of prominent interest in applications. We have to add that the main results obtained in this paper hold under the condition

$$
\mathbb{P}(\alpha<0)>0 .
$$

In this case we still do not have extensive knowledge about the existence of non-constant solutions of the archetypal equation in the class of bounded continuous functions. It is worth noting that every absolutely continuous solution of (1.1) whose derivative belongs to $\mathrm{L}^{1}(\mathbb{R})$ is constant when (1.4) holds (see Theorem 4.5 from [3]). Furthermore we are able to obtain and describe all bounded continuous solutions of the archetypal equation when (1.4) holds only if $|\alpha|=1$ a.s. (see Theorem 2.3 from [4]). Each of them is uniformly continuous. It was also proved in [4] that if $|\alpha| \neq 1$ a.s. and $K \leq 0$, then every bounded and uniformly continuous solution of (1.1) is constant. The problem of the existence or non-existence of non-trivial solutions of (1.1) when (1.4) is true and $K>0$ is still open today.

In the present paper we will prove that, under some mild technical assumption imposed on the measure $\mu$, every bounded continuous solution of (1.1) reaching the global extreme value must be constant. In the proof we make use of the method in [9]. In the third section we prove some results connected with the asymptotical behaviour of bounded continuous solutions of the archetypal equation. 


\section{Solutions attaining the global extreme value}

Let us begin with a simple observation.

Remark 2.1. Note that each constant function is a solution of the archetypal equation since $\mu$ is a probability measure. Furthermore, the linear combination of solutions of (1.1) is still its solution. Hence, studying bounded solutions $\varphi: \mathbb{R} \rightarrow \mathbb{R}$ of (1.1) which attain their global extremum we can assume without loss of generality that $\min \varphi(\mathbb{R})=0$ and $\sup \varphi(\mathbb{R}) \leq 1$.

By the support of a Borel measure $\mu$ on $\mathbb{R}^{n}$, where $n \in \mathbb{N}$, we mean the set supp $\mu$ of all $x \in \mathbb{R}^{n}$ such that each neighborhood of $x$ is of positive measure $\mu$. Observe that $\operatorname{supp} \mu$ is a closed subset of $\mathbb{R}^{n}$. In what follows $\mu$ stands for an arbitrary Borel probability measure on $\mathbb{R}^{2}$.

We prove the following technical lemma which will be our basic tool in the proof of the main theorems.

Lemma 2.2. Let $\varphi: \mathbb{R} \rightarrow[0,+\infty)$ be a continuous solution of equation (1.1) and let $x_{0} \in \mathbb{R}$ be such that $\varphi\left(x_{0}\right)=0$. Then

$$
\varphi\left(c_{p} c_{p-1} \cdot \ldots \cdot c_{1} x_{0}-\sum_{i=1}^{p} c_{p} \cdot \ldots \cdot c_{i} d_{i}\right)=0
$$

for all $p \in \mathbb{N}$ and $\left(c_{1}, d_{1}\right), \ldots,\left(c_{p}, d_{p}\right) \in \operatorname{supp} \mu$.

Proof. Let $p \in \mathbb{N}$ be fixed and assume that $\left(c_{1}, d_{1}\right), \ldots,\left(c_{p}, d_{p}\right) \in \operatorname{supp} \mu$. Applying equality (1.1) to $x_{0}$ we get

$$
0=\varphi\left(x_{0}\right)=\iint_{\mathbb{R}^{2}} \varphi\left(a\left(x_{0}-b\right)\right) \mu(d a, d b) .
$$

Since $\varphi$ is continuous and nonnegative, we have

$$
\varphi\left(a\left(x_{0}-b\right)\right)=0 \text { for all }(a, b) \in \operatorname{supp} \mu .
$$

In particular, we get $\varphi\left(c_{1} x_{0}-c_{1} d_{1}\right)=0$. If we repeat this reasoning $p-1$ times to the points $c_{j} \cdot \ldots \cdot c_{1} x_{0}-\sum_{i=1}^{j} c_{j} \cdot \ldots \cdot c_{i} d_{i}$ and the pairs $\left(c_{j+1}, d_{j+1}\right)$, $j=1, \ldots, p-1$, in turn, we obtain the assertion.

We will also prove a lemma which asserts that some subsets are dense in $\mathbb{R}$. The statement is obvious but it can help to draw attention to the crucial properties of sets which are considered in the proof of Theorem 2.4. We will also present a simple proof of the lemma for the sake of completeness.

Lemma 2.3. Let $t \in \mathbb{R} \backslash\{0\}, v \in(1,+\infty)$ and let $\left(u_{n}\right)_{n \in \mathbb{N}}$ be a sequence of real numbers. Then the set $D=\bigcup_{n=1}^{+\infty} D_{n}$, where

$$
D_{n}:=\left\{u_{n}+\frac{s t}{v^{n}}: s \in \mathbb{Z}\right\} \text { for all } n \in \mathbb{N},
$$

is dense in $\mathbb{R}$. 
Proof. We will consider the case when $t$ is positive. We fix $a, b \in \mathbb{R}$ such that $a<b$ and define the interval $I=(a, b)$. We have to show that $I \cap D$ is nonempty. Let $n_{0} \in \mathbb{N}$ be such that

$$
\frac{t}{v^{n_{0}}}<b-a
$$

Let $s_{0}$ be the greatest number of $\mathbb{Z}$ for which

$$
u_{n_{0}}+\frac{s_{0} t}{v^{n_{0}}} \leq a .
$$

Hence, by the definition of the number $s_{0}$, we have

$$
u_{n_{0}}+\frac{s_{0} t+t}{v^{n_{0}}}>a .
$$

Moreover, using the definition of $s_{0}$ and inequality (2.1) we get

$$
u_{n_{0}}+\frac{s_{0} t+t}{v^{n_{0}}}=u_{n_{0}}+\frac{s_{0} t}{v^{n_{0}}}+\frac{t}{v^{n_{0}}} \leq a+\frac{t}{v^{n_{0}}}<b .
$$

Therefore $u_{n_{0}}+\frac{s_{0} t+t}{v^{n} 0} \in I$. It means that $D$ is dense in $\mathbb{R}$. If $t$ is negative then the proof is similar, so we omit it.

Theorem 2.4. If $\mu(((-\infty, 0) \backslash\{-1\}) \times \mathbb{R})>0$ and there exists $b \in \mathbb{R} \backslash\{0\}$ such that $(1, b) \in$ supp $\mu$, then every bounded continuous solution of equation (1.1) attaining its global extremum is constant.

Remark 2.5. It is worth mentioning that the assumption of the boundedness of $\varphi$ in Theorem 2.4 is essential only in the case $\mu(\mathbb{Z} \times \mathbb{R})=1$.

Proof. The pair $(1, b)$ will be denoted by $\left(a_{0}, b_{0}\right)$ from this point. Take any bounded continuous solution $\varphi: \mathbb{R} \rightarrow \mathbb{R}$ of (1.1) attaining its global extremum. In view of Remark 2.1 we can assume that $\varphi: \mathbb{R} \rightarrow[0,1]$ and there exists $x_{0} \in \mathbb{R}$ such that $\varphi\left(x_{0}\right)=0$. Since $\varphi$ is continuous, it is sufficient to show that $\varphi$ takes value 0 on some dense subset $D$ of the real line. The proof is split into the three general cases and in each of them we will describe how to obtain the desired dense set. We shall examine each of the following situations:
I. $\operatorname{supp} \mu \cap((\mathbb{R} \backslash \mathbb{Q}) \times \mathbb{R}) \neq \emptyset$,
II. $\operatorname{supp} \mu \cap((\mathbb{Q} \backslash \mathbb{Z}) \times \mathbb{R}) \neq \emptyset$,
III. $\operatorname{supp} \mu \subseteq \mathbb{Z} \times \mathbb{R}$.

Furthermore we distinguish two complementary subcases for both case I and case II.

I.A. There exist $a \in(\mathbb{R} \backslash \mathbb{Q}) \cap(-\infty, 0)$ and $b \in \mathbb{R}$ such that $(a, b) \in \operatorname{supp} \mu$.

I.B. If $(a, b) \in \operatorname{supp} \mu$ and $a \in \mathbb{R} \backslash \mathbb{Q}$, then $a$ is positive.

II.A. There exist $a \in(\mathbb{Q} \backslash \mathbb{Z}) \cap(-\infty, 0)$ and $b \in \mathbb{R}$ such that $(a, b) \in \operatorname{supp} \mu$.

II.B. If $(a, b) \in \operatorname{supp} \mu$ and $a \in \mathbb{Q} \backslash \mathbb{Z}$, then $a$ is positive. 
In summary, we obtain the five cases (I.A, I.B, II.A, II.B and III) and note that the measure $\mu$ fulfills at least one of them. We shall prove that in each one the function $\varphi$ is constant.

I.A. Assume that there exists $(a, b) \in \operatorname{supp} \mu$ such that $a \in(-\infty, 0) \backslash \mathbb{Q}$. Put $\left(a_{1}, b_{1}\right):=(a, b)$ and remember that $a_{0}=1$ and $b_{0} \neq 0$. Fix also arbitrary $k, l \in \mathbb{N}$. If we apply Lemma 2.2 with $\left(c_{i}, d_{i}\right)=\left(a_{0}, b_{0}\right)$, where $i=1,2, \ldots, k$, then we obtain the equality $\varphi\left(x_{0}-k b_{0}\right)=0$ since $a_{0}=1$. Applying equality (1.1) for $x_{0}-k b_{0}$ one can deduce that

$$
\varphi\left(a_{1}\left(x_{0}-b_{1}\right)-a_{1} k b_{0}\right)=0 .
$$

Finally, if we use Lemma 2.2 with $\left(c_{i}, d_{i}\right)=\left(a_{0}, b_{0}\right)$, where $i=1,2, \ldots, l$, to the point $a_{1}\left(x_{0}-b_{1}\right)-a_{1} k b_{0}$, then we will get

$$
\varphi\left(a_{1}\left(x_{0}-b_{1}\right)-\left(k a_{1}+l\right) b_{0}\right)=0 .
$$

The Kronecker density theorem [7, Chapter XXIII] asserts that the set

$$
\left\{k a_{1}+l: k, l \in \mathbb{N}\right\}
$$

is dense in $\mathbb{R}$. Hence

$$
\left\{a_{1}\left(x_{0}-b_{1}\right)-\left(k a_{1}+l\right) b_{0}: k, l \in \mathbb{N}\right\}
$$

is also dense as $b_{0} \neq 0$. This implies that $\varphi$ is constant and the proof is complete in this subcase.

I.B. This case means that if $(a, b) \in \operatorname{supp} \mu$ and $a$ is negative, then $a \in$ $\mathbb{Q}$. Since $\mu((-\infty, 0) \times \mathbb{R})>0$ there exists $(a, b) \in \operatorname{supp} \mu$ with $a<0$. Put $\left(a_{1}, b_{1}\right):=(a, b)$. Then $a_{1} \in \mathbb{Q}$. Take also $\left(a_{2}, b_{2}\right) \in \operatorname{supp} \mu$ with $a_{2} \in \mathbb{R} \backslash \mathbb{Q}$. Then $a_{2}>0$. As before we obtain the equality $\varphi\left(a_{1}\left(x_{0}-b_{1}\right)-a_{1} k b_{0}\right)=0$, where $k \in \mathbb{N}$ is fixed. In the next step, applying equality (1.1) to $a_{1}\left(x_{0}-b_{1}\right)-$ $a_{1} k b_{0}$, we get

$$
\varphi\left(a_{1} a_{2}\left(x_{0}-b_{1}\right)-a_{2} b_{2}-a_{1} a_{2} k b_{0}\right)=0 .
$$

Fix an arbitrary $l \in \mathbb{N}$. Finally, we apply Lemma 2.2 with $\left(c_{i}, d_{i}\right)=\left(a_{0}, b_{0}\right)$ for $i=1, \ldots, l$ to the point $a_{1} a_{2}\left(x_{0}-b_{1}\right)-a_{2} b_{2}-a_{1} a_{2} k b_{0}$. As a result we have

$$
\varphi\left(a_{1} a_{2}\left(x_{0}-b_{1}\right)-a_{2} b_{2}-\left(l+a_{1} a_{2} k\right) b_{0}\right)=0 .
$$

Note that $a_{1} a_{2}$ is a negative irrational number, so again using the Kronecker density theorem we have constructed a dense subset $D$ of $\mathbb{R}$ while $k, l$ runs through $\mathbb{N}$. The proof is complete in this case.

II. Now we assume that $\operatorname{supp} \mu \cap((\mathbb{Q} \backslash \mathbb{Z}) \times \mathbb{R}) \neq \emptyset$. Since we are done in case I we may assume that $\operatorname{supp} \mu \subseteq \mathbb{Q} \times \mathbb{R}$. Let $A \subseteq \mathbb{Q}$ be the smallest set such that $\operatorname{supp} \mu \subseteq A \times \mathbb{R}$, i.e.

$$
A=\{a \in \mathbb{Q}:(a, b) \in \operatorname{supp} \mu \text { for some real } b\} .
$$

Then there is a set $I \subseteq \mathbb{N} \cup\{0\}$ and an injective sequence $\left(a_{i}\right)_{i \in I}$ such that $A=\left\{a_{i}: i \in I\right\}$. Moreover, we can take $I=\{0,1, \ldots, n-1\}$ if $A$ has exactly $n$ elements and $I=\mathbb{N} \cup\{0\}$ when $A$ is infinite. Observe that the condition 
$\operatorname{supp} \mu \cap((\mathbb{Q} \backslash \mathbb{Z}) \times \mathbb{R}) \neq \emptyset$, assumed in the present case II, means that $A \backslash \mathbb{Z} \neq \emptyset$. For every $i \in I$ we also define a Borel measure $\mu_{i}$ by the equality

$$
\mu_{i}(B)=\mu\left(\left\{a_{i}\right\} \times B\right) \text { for each Borel } B \subseteq \mathbb{R} .
$$

Note that for any $i \in I$ we have $\mu_{i}(\mathbb{R}) \in(0,1)$ and $\sum_{i \in I} \mu_{i}(\mathbb{R})=1$. Moreover, equation (1.1) can be rewritten in the form

$$
\varphi(x)=\sum_{i \in I} \int_{\mathbb{R}} \varphi\left(a_{i}(x-b)\right) \mu_{i}(d b) .
$$

II.A. In this case there exists $i \in I$ such that $a_{i}$ is negative and noninteger. First of all remember that $a_{0}=1$ and $b_{0} \neq 0$. Without loss of generality we may assume that $i=1$. In other words $a_{1} \in(-\infty, 0) \cap(\mathbb{Q} \backslash \mathbb{Z})$. Then there exist coprimes $q, q_{0} \in \mathbb{Z}$ such that $a_{1}=q / q_{0}$ and $q<0$. The definitions of $q, q_{0}$ and $a_{1}$ imply that $q_{0} \geq 2$. We fix also any $b_{1} \in \mathbb{R}$ for which $\left(a_{1}, b_{1}\right) \in \operatorname{supp} \mu$. Define the sequence $\left(D_{n}\right)_{n \in \mathbb{N}}$ of sets putting

$$
D_{n}:=\left\{a_{1}^{n} x_{0}-\sum_{i=1}^{n} a_{1}^{i} b_{1}-\frac{s b_{0}}{q_{0}^{n}}: s \in \mathbb{Z}\right\}
$$

and let

$$
D:=\bigcup_{n=1}^{+\infty} D_{n}
$$

Since $b_{0} \neq 0$ and $q_{0}>1$, the density of the set $D$ follows from Lemma 2.3.

Our goal is to prove that the function $\varphi$ vanishes on $D$. We shall use mathematical induction with respect to $n$. In the first step we show that $\varphi_{\mid D_{1}}=0$, i.e.

$$
\varphi\left(a_{1}\left(x_{0}-b_{1}\right)-\frac{s b_{0}}{q_{0}}\right)=0 \text { for all } s \in \mathbb{Z} .
$$

Fix any $k, l \in \mathbb{N} \cup\{0\}$. In the beginning note that we can obtain the equality

$$
0=\varphi\left(a_{1}\left(x_{0}-b_{1}\right)-a_{1} k b_{0}\right)=\varphi\left(a_{1}\left(x_{0}-b_{1}\right)-\frac{q k}{q_{0}} b_{0}\right)
$$

in the same way as in point I.A. If we apply Lemma 2.2 with $\left(c_{j}, d_{j}\right)=\left(a_{0}, b_{0}\right)$, where $j=1,2, \ldots, l$, to the point $a_{1}\left(x_{0}-b_{1}\right)-\frac{q k}{q_{0}} b_{0}$, then we will get

$$
\varphi\left(a_{1}\left(x_{0}-b_{1}\right)-l b_{0}-\frac{q k}{q_{0}} b_{0}\right)=\varphi\left(a_{1}\left(x_{0}-b_{1}\right)-\frac{q_{0} l+q k}{q_{0}} b_{0}\right)=0 .
$$

Note that the expression $q_{0} l+q k$ runs through the whole $\mathbb{Z}$, while $k, l \in \mathbb{N} \cup\{0\}$, since $q$ and $q_{0}$ are coprime and of different signs. Therefore equality (2.3) implies that

$$
\varphi(x)=0 \text { for every } x \in D_{1} \text {. }
$$


Let $n \in \mathbb{N}$ be fixed and assume that $\varphi_{\mid D_{n}}=0$. We prove that $\varphi_{\mid D_{n+1}}=0$. Take any $s \in \mathbb{Z}$, put

$$
r=a_{1}^{n+1} x_{0}-\sum_{i=1}^{n+1} a_{1}^{i} b_{1}-\frac{s b_{0}}{q_{0}^{n+1}}
$$

and observe that $r \in D_{n+1}$. Since $q$ and $q_{0}$ are coprime and of different signs, we can find $k, l \in \mathbb{N} \cup\{0\}$ such that $q_{0}^{n+1} l+q k=s$. If we use the induction hypothesis and equality (2.2), then we will get

$$
\begin{aligned}
0 & =\varphi\left(a_{1}^{n} x_{0}-\sum_{i=1}^{n} a_{1}^{i} b_{1}-\frac{k b_{0}}{q_{0}^{n}}\right) \\
& =\sum_{j \in I} \int_{\mathbb{R}} \varphi\left(a_{j}\left(a_{1}^{n} x_{0}-\sum_{i=1}^{n} a_{1}^{i} b_{1}-\frac{k b_{0}}{q_{0}^{n}}-b\right)\right) \mu_{j}(d b),
\end{aligned}
$$

and thus, as $\varphi$ is non-negative,

$$
\int_{\mathbb{R}} \varphi\left(a_{j}\left(a_{1}^{n} x_{0}-\sum_{i=1}^{n} a_{1}^{i} b_{1}-\frac{k b_{0}}{q_{0}^{n}}-b\right)\right) \mu_{j}(d b)=0 \text { for all } j \in I .
$$

In particular, for $j=1$ we have

$$
\int_{\mathbb{R}} \varphi\left(a_{1}\left(a_{1}^{n} x_{0}-\sum_{i=1}^{n} a_{1}^{i} b_{1}-\frac{k b_{0}}{q_{0}^{n}}-b\right)\right) \mu_{1}(d b)=0 .
$$

The function $\varphi$ is non-negative and continuous. Hence

$$
\varphi\left(a_{1}\left(a_{1}^{n} x_{0}-\sum_{i=1}^{n} a_{1}^{i} b_{1}-\frac{k b_{0}}{q_{0}^{n}}-b\right)\right)=0 \text { for every } b \in \operatorname{supp} \mu_{1} .
$$

Taking $b=b_{1}$ in the above equality we get

$$
\varphi\left(a_{1}^{n+1} x_{0}-\sum_{i=1}^{n} a_{1}^{i+1} b_{1}-a_{1} b_{1}-a_{1} \frac{k b_{0}}{q_{0}^{n}}\right)=0 .
$$

Thus, using the representation $a_{1}=q / q_{0}$, we come to the equality

$$
\varphi\left(a_{1}^{n+1} x_{0}-\sum_{i=1}^{n+1} a_{1}^{i} b_{1}-\frac{q k b_{0}}{q_{0}^{n+1}}\right)=0 .
$$

Recall that $a_{0}=1$ and the numbers $k$ and $l$ were chosen so that the sum $q_{0} l^{n+1}+q k$ is equal to $s$. If we apply Lemma 2.2 with $\left(c_{j}, d_{j}\right)=\left(a_{0}, b_{0}\right)$, where $j=1,2, \ldots, l$, to the point $a_{1}^{n+1} x_{0}-\sum_{i=1}^{n+1} a_{1}^{i} b_{1}-\frac{q k b_{0}}{q_{0}^{n+1}}$, then we will get

$$
\varphi\left(a_{1}^{n+1} x_{0}-\sum_{i=1}^{n+1} a_{1}^{i} b_{1}-\frac{q k b_{0}}{q_{0}^{n+1}}-l b_{0}\right)=0,
$$


that is

$$
\varphi\left(a_{1}^{n+1} x_{0}-\sum_{i=1}^{n+1} a_{1}^{i} b_{1}-\frac{q k+q_{0}^{n+1} l}{q_{0}^{n+1}} b_{0}\right)=0 .
$$

The above equality means that $\varphi(r)=0$. Since $r$ was an arbitrary element of $D_{n+1}$, we have proven that

$$
\varphi(x)=0 \text { for every } x \in D_{n+1} .
$$

Consequently, by mathematical induction, we get $\varphi_{\mid D}=0$ and the proof has been completed in case II.A.

II.B. In this case $A \backslash \mathbb{Z} \neq \emptyset$ and each negative element of $A$ is an integer. Recall that we have $a_{0}=1$ and $b_{0} \neq 0$. We know that $\mu((-\infty, 0) \times \mathbb{R})>0$. This condition implies that we can choose $i \in I$ such that $a_{i} \in(-\infty, 0)$. All such numbers are, in view of the assumptions of case II.B, integers. Without loss of generality we may assume that $i=1$. Then $a_{1} \in \mathbb{Z}$. Take an arbitrary $b_{1} \in \operatorname{supp} \mu_{1}$. Since $\emptyset \neq A \backslash \mathbb{Z} \subseteq \mathbb{Q} \backslash \mathbb{Z}$ we may assume that $a_{2} \in \mathbb{Q} \backslash \mathbb{Z}$. Take any $b_{2} \in \operatorname{supp} \mu_{2}$. Then $a_{2}$ must be positive. We also define a parameter $m \in \mathbb{N}$ such that $a_{1} a_{2}^{m} \in \mathbb{Q} \backslash \mathbb{Z}$ since it may happen that $a_{1} a_{2} \in \mathbb{Z}$. There exist coprimes $q, q_{0} \in \mathbb{Z}$ for which $a_{1} a_{2}^{m}=q / q_{0}$ and $q<0$. Then $q_{0} \geq 2$. The proof in this case is analogous to that of the previous subcase. This time a sequence of sets $\left(D_{n}\right)_{n \in \mathbb{N}}$ will be defined by

$$
D_{n}:=\left\{\left(a_{1} a_{2}^{m}\right)^{n} x_{0}-\sum_{i=1}^{n}\left(a_{1} a_{2}^{m}\right)^{i} b_{1}-\sum_{i=0}^{n-1} \sum_{j=1}^{m} a_{1}^{i} a_{2}^{i m+j} b_{2}-\frac{s b_{0}}{q_{0}^{n}}: s \in \mathbb{Z}\right\}
$$

and again we put

$$
D=\bigcup_{n=1}^{+\infty} D_{n}
$$

We have $b_{0} \neq 0$ and $q_{0}>1$. Define a sequence $\left(u_{n}\right)_{n \in \mathbb{N}}$ by

$$
u_{n}=\left(a_{1} a_{2}^{m}\right)^{n} x_{0}-\sum_{i=1}^{n}\left(a_{1} a_{2}^{m}\right)^{i} b_{1}-\sum_{i=0}^{n-1} \sum_{j=1}^{m} a_{1}^{i} a_{2}^{i m+j} b_{2} .
$$

Then, in view of Lemma 2.3, we will get the density of $D$. Again we shall use mathematical induction to prove that $\varphi$ is constant on $D$. First of all we are going to show that $\varphi_{\mid D_{1}}=0$. As previously we start with the equality

$$
\varphi\left(a_{1}\left(x_{0}-b_{1}\right)-a_{1} k b_{0}\right)=0
$$

where $k \in \mathbb{N} \cup\{0\}$ is fixed. If we apply Lemma 2.2 with $\left(c_{j}, d_{j}\right)=\left(a_{2}, b_{2}\right)$, where $j=1,2, \ldots, m$, to the point $a_{1}\left(x_{0}-b_{1}\right)-a_{1} k b_{0}$, then we will get

$$
\varphi\left(a_{1} a_{2}^{m}\left(x_{0}-b_{1}\right)-\sum_{i=1}^{m} a_{2}^{i} b_{2}-a_{1} a_{2}^{m} k b_{0}\right)=0 .
$$


Since $a_{1} a_{2}^{m}=q / q_{0}$, we have

$$
\varphi\left(a_{1} a_{2}^{m}\left(x_{0}-b_{1}\right)-\sum_{i=1}^{m} a_{2}^{i} b_{2}-\frac{q k}{q_{0}} b_{0}\right)=0 .
$$

Fix any $l \in \mathbb{N} \cup\{0\}$. If we use Lemma 2.2 with $\left(c_{j}, d_{j}\right)=\left(a_{0}, b_{0}\right)$, where $j=1, \ldots, l$, to the point from the above equality, then we will obtain

$$
\varphi\left(a_{1} a_{2}^{m}\left(x_{0}-b_{1}\right)-\sum_{i=1}^{m} a_{2}^{i} b_{2}-\frac{q k}{q_{0}} b_{0}-l b_{0}\right)=0,
$$

that is

$$
\varphi\left(a_{1} a_{2}^{m}\left(x_{0}-b_{1}\right)-\sum_{i=1}^{m} a_{2}^{i} b_{2}-\frac{q k+q_{0} l}{q_{0}} b_{0}\right)=0 .
$$

Note that $q$ and $q_{0}$ are coprime and they have different signs which implies that the expression $q k+q_{0} l$ can take any integer value when $k, l$ run through $\mathbb{N} \cup\{0\}$. Hence

$$
\varphi(x)=0 \text { for every } x \in D_{1} .
$$

Fix $n \in \mathbb{N}$ and assume that $\varphi_{\mid D_{n}}=0$. We will show the equality $\varphi_{\mid D_{n+1}}=0$. Take any $s \in \mathbb{Z}$ and put

$$
r:=\left(a_{1} a_{2}^{m}\right)^{n+1} x_{0}-\sum_{i=1}^{n+1}\left(a_{1} a_{2}^{m}\right)^{i} b_{1}-\sum_{i=0}^{n} \sum_{j=1}^{m} a_{1}^{i} a_{2}^{i m+j} b_{2}-\frac{s b_{0}}{q_{0}^{n+1}} .
$$

Note that $r$ is a member of the set $D_{n+1}$. We show that $\varphi(r)=0$. As in the proof of case II.A we fix $k, l \in \mathbb{N} \cup\{0\}$ such that $l q_{0}^{n+1}+k q=s$. Such a choice is possible since $q$ and $q_{0}$ are coprime and $q<0<q_{0}$. Moreover, we have

$$
\varphi\left(\left(a_{1} a_{2}^{m}\right)^{n} x_{0}-\sum_{i=1}^{n}\left(a_{1} a_{2}^{m}\right)^{i} b_{1}-\sum_{i=0}^{n-1} \sum_{j=1}^{m} a_{1}^{i} a_{2}^{i m+j} b_{2}-\frac{k b_{0}}{q_{0}^{n}}\right)=0
$$

since the argument from the above equality belongs to $D_{n}$. Applying equality (2.2) to the point from the last equality, for every $i \in I$ and $b \in \operatorname{supp} \mu_{i}$ we get

$$
\varphi\left(a_{i}\left(\left(a_{1} a_{2}^{m}\right)^{n} x_{0}-\sum_{i=1}^{n}\left(a_{1} a_{2}^{m}\right)^{i} b_{1}-b-\sum_{i=0}^{n-1} \sum_{j=1}^{m} a_{1}^{i} a_{2}^{i m+j} b_{2}-\frac{k b_{0}}{q_{0}^{n}}\right)\right)=0 .
$$

In particular for $i=1$ and $b=b_{1}$, we have

$$
\varphi\left(a_{1}\left(a_{1} a_{2}^{m}\right)^{n} x_{0}-a_{1} \sum_{i=1}^{n}\left(a_{1} a_{2}^{m}\right)^{i} b_{1}-a_{1} b_{1}-\sum_{i=0}^{n-1} \sum_{j=1}^{m} a_{1}^{i+1} a_{2}^{i m+j} b_{2}-a_{1} \frac{k b_{0}}{q_{0}^{n}}\right)=0 .
$$


If we apply Lemma 2.2 with $\left(c_{j}, d_{j}\right)=\left(a_{2}, b_{2}\right)$, where $j=1,2, \ldots, m$, to the argument from the previous equality and use the identities

$$
a_{1} a_{2}^{m} \sum_{i=1}^{n}\left(a_{1} a_{2}^{m}\right)^{i} b_{1}+a_{1} a_{2}^{m} b_{1}=\sum_{i=1}^{n+1}\left(a_{1} a_{2}^{m}\right)^{i} b_{1}
$$

and

$$
a_{2}^{m} \sum_{i=0}^{n-1} \sum_{j=1}^{m} a_{1}^{i+1} a_{2}^{i m+j} b_{2}+\sum_{j=1}^{m} a_{2}^{j} b_{2}=\sum_{i=0}^{n} \sum_{j=1}^{m} a_{1}^{i} a_{2}^{i m+j} b_{2}
$$

then we get

$$
\varphi\left(\left(a_{1} a_{2}^{m}\right)^{n+1} x_{0}-\sum_{i=1}^{n+1}\left(a_{1} a_{2}^{m}\right)^{i} b_{1}-\sum_{i=0}^{n} \sum_{j=1}^{m} a_{1}^{i} a_{2}^{i m+j} b_{2}-a_{1} a_{2}^{m} \frac{k b_{0}}{q_{0}^{n}}\right)=0 .
$$

Since $a_{1} a_{2}^{m}=q / q_{0}$, the last equality can be rewritten as

$$
\varphi\left(\left(a_{1} a_{2}^{m}\right)^{n+1} x_{0}-\sum_{i=1}^{n+1}\left(a_{1} a_{2}^{m}\right)^{i} b_{1}-\sum_{i=0}^{n} \sum_{j=1}^{m} a_{1}^{i} a_{2}^{i m+j} b_{2}-\frac{q k b_{0}}{q_{0}^{n+1}}\right)=0 .
$$

Recall that $a_{0}=1$. If we use Lemma 2.2 with $\left(c_{j}, d_{j}\right)=\left(a_{0}, b_{0}\right)$, where $j=$ $1,2, \ldots, l$, to the number from the above equality, then we will get

$$
\begin{aligned}
0 & =\varphi\left(\left(a_{1} a_{2}^{m}\right)^{n+1} x_{0}-\sum_{i=1}^{n+1}\left(a_{1} a_{2}^{m}\right)^{i} b_{1}-\sum_{i=0}^{n} \sum_{j=1}^{m} a_{1}^{i} a_{2}^{i m+j} b_{2}-\frac{q k b_{0}}{q_{0}^{n+1}}-l b_{0}\right) \\
& =\varphi\left(\left(a_{1} a_{2}^{m}\right)^{n+1} x_{0}-\sum_{i=1}^{n+1}\left(a_{1} a_{2}^{m}\right)^{i} b_{1}-\sum_{i=0}^{n} \sum_{j=1}^{m} a_{1}^{i} a_{2}^{i m+j} b_{2}-\frac{l q_{0}^{n+1}+k q}{q_{0}^{n+1}} b_{0}\right) .
\end{aligned}
$$

We have chosen the numbers $k$ and $l$ in such a way that $l q_{0}^{n+1}+k q=s$. Hence

$$
\varphi\left(\left(a_{1} a_{2}^{m}\right)^{n+1} x_{0}-\sum_{i=1}^{n+1}\left(a_{1} a_{2}^{m}\right)^{i} b_{1}-\sum_{i=0}^{n} \sum_{j=1}^{m} a_{1}^{i} a_{2}^{i m+j} b_{2}-\frac{s b_{0}}{q_{0}^{n+1}}\right)=0,
$$

i.e. $\varphi(r)=0$. This equality implies that $\varphi_{\mid D_{n+1}}=0$ since $r$ was taken arbitrarily. In view of the mathematical induction we have $\varphi_{\mid D}=0$ and the proof is complete in case II.B.

III. We shall consider the last case when $\operatorname{supp} \mu \subset \mathbb{Z} \times \mathbb{R}$, that is $A \subseteq \mathbb{Z}$. Recall that $a_{0}=1$ and $b_{0} \neq 0$. Since $\mu(((-\infty, 0) \backslash\{-1\}) \times \mathbb{R})>0$ we can choose $i \in I$ such that $a_{i}$ is a negative integer different from -1 . Without loss of generality we may assume that $i=1$. Fix any $b_{1} \in \operatorname{supp} \mu_{1}$. We define 
a sequence of sets $\left(D_{n}\right)_{n \in \mathbb{N} \cup\{0\}}$ setting

$$
\begin{aligned}
D_{n}:= & \left\{e_{p} \ldots e_{1} x_{0}-\sum_{i=1}^{p} e_{p} \ldots e_{i} f_{i}-\frac{s b_{0}}{a_{1}^{n}}: p \in \mathbb{N}, s \in \mathbb{Z},\right. \\
& \left.\left(e_{1}, f_{1}\right), \ldots,\left(e_{p}, f_{p}\right) \in \operatorname{supp} \mu \text { and } \exists_{i_{0} \in\{1,2, \ldots, p\}} e_{i_{0}}=a_{1}\right\},
\end{aligned}
$$

and let

$$
D:=\bigcup_{n=0}^{+\infty} D_{n}
$$

Note that $D_{n}$ contains as a subset the set

$$
\left\{a_{1}\left(x_{0}-b_{1}\right)-\frac{s b_{0}}{a_{1}^{n}}: s \in \mathbb{Z}\right\} .
$$

We have $b_{0} \neq 0$ and $a_{1}^{2}>1$. Lemma 2.3 asserts that the set

$$
\bigcup_{n=0}^{+\infty}\left\{a_{1}\left(x_{0}-b_{1}\right)-\frac{s b_{0}}{a_{1}^{2 n}}: s \in \mathbb{Z}\right\}
$$

is dense in $\mathbb{R}$. Hence also $D$ is dense. Moreover, now we will show that the sequence $\left(D_{n}\right)_{n \in \mathbb{N} \cup\{0\}}$ has the following properties:

P1. If $r \in D_{n}$ for some $n \in \mathbb{N}$ and $i \in I \backslash\{1\}$, then

$$
a_{i}(r-b) \in D_{n} \text { for every } b \in \operatorname{supp} \mu_{i} .
$$

P2. If $r \in D_{n}$ for some $n \in \mathbb{N}$, then

$$
a_{1}(r-b) \in D_{n-1} \text { for every } b \in \operatorname{supp} \mu_{1} .
$$

Fix $n \in \mathbb{N}$ and take any $r \in D_{n}$. Let $p \in \mathbb{N}$ and $\left(e_{1}, f_{1}\right), \ldots,\left(e_{p}, f_{p}\right) \in \operatorname{supp} \mu$ be such that $a_{1}$ is one of $e_{1}, \ldots, e_{p}$ and

$$
r=e_{p} \ldots e_{1} x_{0}-\sum_{i=1}^{p} e_{p} \ldots e_{i} f_{i}-\frac{s b_{0}}{a_{1}^{n}}
$$

with some $s \in \mathbb{Z}$. Take an arbitrary $i \in I$ and $b \in \operatorname{supp} \mu_{i}$. Then $\left(a_{i}, b\right) \in \operatorname{supp} \mu$ and

$$
a_{i}(r-b)=a_{i} e_{p} \ldots e_{1} x_{0}-a_{i} \sum_{i=1}^{p} e_{p} \ldots e_{i} f_{i}-a_{i} b-\frac{a_{i} s b_{0}}{a_{1}^{n}}
$$

that is

$$
a_{i}(r-b)=e_{p+1} e_{p} \ldots e_{1} x_{0}-\sum_{i=1}^{p+1} e_{p+1} \ldots e_{i} f_{i}-\frac{a_{i} s b_{0}}{a_{1}^{n}},
$$

where $e_{p+1}:=a_{i}$ and $f_{p+1}:=b$. Since $A \subseteq \mathbb{Z}$ we have $a_{i} s \in \mathbb{Z}$. Consequently, $a_{i}(r-b) \in D_{n}$. If, in addition, $i=1$, then $a_{i}(r-b)=a_{1}(r-b) \in D_{n-1}$. 
Now we are going to show that the function $\varphi$ is constant on $D$. As before we will use mathematical induction and start with showing that $\varphi_{\mid D_{0}}=0$. Let us recall that

$$
\begin{aligned}
D_{0} & =\left\{e_{p} \ldots e_{1} x_{0}-\sum_{i=1}^{p} e_{p} \ldots e_{i} f_{i}-s b_{0}: p \in \mathbb{N}, s \in \mathbb{Z},\right. \\
& \left.\left(e_{1}, f_{1}\right), \ldots,\left(e_{p}, f_{p}\right) \in \operatorname{supp} \mu \text { and } \exists_{i_{0} \in\{1,2, \ldots, p\}} e_{i_{0}}=a_{1}\right\} .
\end{aligned}
$$

Fix any $p \in \mathbb{N}, s \in \mathbb{Z}$ and $\left(e_{1}, f_{1}\right), \ldots,\left(e_{p}, f_{p}\right) \in \operatorname{supp} \mu$ such that $e_{i_{0}}=a_{1}$ for some $i_{0} \in\{1,2, \ldots, p\}$. In the first step we show the equality

$$
\varphi\left(e_{i_{0}} \ldots e_{1} x_{0}-\sum_{i=1}^{i_{0}} e_{i_{0}} \ldots e_{i} f_{i}-s b_{0}\right)=0 .
$$

Applying Lemma 2.2 with $\left(c_{j}, d_{j}\right)=\left(e_{j}, f_{j}\right)$, where $j=1,2, \ldots, i_{0}-1$, to the point $x_{0}$ we get

$$
\varphi\left(e_{i_{0}-1} \ldots e_{1} x_{0}-\sum_{i=1}^{i_{0}-1} e_{i_{0}-1} \ldots e_{i} f_{i}\right)=0 .
$$

Fix arbitrary $k \in \mathbb{N} \cup\{0\}$ and remember that $a_{0}=1$. If we use Lemma 2.2 with $\left(c_{j}, d_{j}\right)=\left(a_{0}, b_{0}\right)$, where $j=1,2, \ldots, k$, to the point from the above equality, then we obtain

$$
\varphi\left(e_{i_{0}-1} \ldots e_{1} x_{0}-\sum_{i=1}^{i_{0}-1} e_{i_{0}-1} \ldots e_{i} f_{i}-k b_{0}\right)=0 .
$$

Putting $x=e_{i_{0}-1} \ldots e_{1} x_{0}-\sum_{i=1}^{i_{0}-1} e_{i_{0}-1} \ldots e_{i} f_{i}-k b_{0}$ in equality (2.2) we have

$$
\varphi\left(a_{i}(x-b)\right)=0 \text { for every } i \in I \text { and } b \in \operatorname{supp} \mu_{i} .
$$

If we put $a_{i}=e_{i_{0}}$ and $b=f_{i_{0}}$ in the last equality, then we come to

$$
\varphi\left(e_{i_{0}} \ldots e_{1} x_{0}-\sum_{i=1}^{i_{0}} e_{i_{0}} \ldots e_{i} f_{i}-e_{i_{0}} k b_{0}\right)=0 .
$$

Let $l \in \mathbb{N} \cup\{0\}$ be arbitrarily fixed. Using Lemma 2.2 with $\left(c_{j}, d_{j}\right)=\left(a_{0}, b_{0}\right)$, where $j=1,2, \ldots, l$, to the point from the previous equality we get

$$
\varphi\left(e_{i_{0}} \ldots e_{1} x_{0}-\sum_{i=1}^{i_{0}} e_{i_{0}} \ldots e_{i} f_{i}-\left(l+e_{i_{0}} k\right) b_{0}\right)=0 .
$$

Since $e_{i_{0}}$ is a negative integer and $k, l \in \mathbb{N} \cup\{0\}$, the expression $l+e_{i_{0}} k$ can attain any integer value. In particular, we can find $k, l \in \mathbb{N} \cup\{0\}$ such that $l+e_{i_{0}} k=s$. This means that equality (2.4) holds. 
We use equality (2.4) to prove that

$$
\varphi\left(e_{p} \ldots e_{1} x_{0}-\sum_{i=1}^{p} e_{p} \ldots e_{i} f_{i}-s b_{0}\right)=0 .
$$

First note that in the case $i_{0}=p$ equality (2.4) reduces to (2.5). Therefore we assume that $i_{0}<p$. Since $e_{i_{0}+1}, \ldots, e_{p} \in \mathbb{Z}$, we can find $\tilde{s} \in \mathbb{Z}$ and $k \in \mathbb{N} \cup\{0\}$ such that $s=k+e_{p} \ldots e_{i_{0}+1} \tilde{s}$. If we use Lemma 2.2 with $\left(c_{j}, d_{j}\right)=\left(e_{i_{0}+j}, f_{i_{0}+j}\right)$, where $j=1,2, \ldots, p-i_{0}$, to the point $e_{i_{0}} \ldots e_{1} x_{0}-\sum_{i=1}^{i_{0}} e_{i_{0}} \ldots e_{1} f_{i}-\tilde{s} b_{0}$, then we will get

$$
\varphi\left(e_{p} \ldots e_{1} x_{0}-\sum_{i=1}^{p} e_{p} \ldots e_{i} f_{i}-e_{p} \ldots e_{i_{0}+1} \tilde{s} b_{0}\right)=0 .
$$

Applying Lemma 2.2 with $\left(c_{j}, d_{j}\right)=\left(a_{0}, b_{0}\right)$, where $j=1,2, \ldots, k$, to the point from the last equality we come to

$$
\varphi\left(e_{p} \ldots e_{1} x_{0}-\sum_{i=1}^{p} e_{p} \ldots e_{i} f_{i}-\left(k+e_{p} \ldots e_{i_{0}+1} \tilde{s}\right) b_{0}\right)=0 .
$$

Putting $s=k+e_{p} \ldots e_{i_{0}+1} \tilde{s}$, we get

$$
\varphi\left(e_{p} \ldots e_{1} x_{0}-\sum_{i=1}^{p} e_{p} \ldots e_{i} f_{i}-s b_{0}\right)=0 .
$$

Hence $\varphi_{\mid D_{0}}=0$.

Now assume that $\varphi_{\mid D_{n}}=0$ for some $n \in \mathbb{N} \cup\{0\}$. We prove that $\varphi_{\mid D_{n+1}}=0$. Let $r \in D_{n+1}$ be fixed and put $J:=I \backslash\{1\}$. Then property P2 asserts that $a_{1}(r-b) \in D_{n}$ for every $b \in \operatorname{supp} \mu_{1}$. Hence using equality (2.2) and the inductive hypothesis we get

$$
\begin{aligned}
\varphi(r) & =\sum_{i \in I} \int_{\mathbb{R}} \varphi\left(a_{i}(r-b)\right) \mu_{i}(d b) \\
& =\sum_{i \in J} \int_{\mathbb{R}} \varphi\left(a_{i}(r-b)\right) \mu_{i}(d b)+\int_{\mathbb{R}} \varphi\left(a_{1}(r-b)\right) \mu_{1}(d b) \\
& =\sum_{i \in J} \int_{\mathbb{R}} \varphi\left(a_{i}(r-b)\right) \mu_{i}(d b)+0=\sum_{i \in J} \int_{\mathbb{R}} \varphi\left(a_{i}(r-b)\right) \mu_{i}(d b) .
\end{aligned}
$$

The function $\varphi$ is bounded above by 1 . Therefore

$$
\varphi(r)=\sum_{i \in J} \int_{\mathbb{R}} \varphi\left(a_{i}(r-b)\right) \mu_{i}(d b) \leq \sum_{i \in J} \int_{\mathbb{R}} \mu_{i}(d b)=\sum_{i \in J} \mu_{i}(\mathbb{R})=1-\mu_{1}(\mathbb{R}) .
$$

If we use equation (2.2) for every $i \in J$, then we will get

$$
\int_{\mathbb{R}} \varphi\left(a_{i}\left(r-b_{1}\right)\right) \mu_{i}\left(d b_{1}\right)=\int_{\mathbb{R}}\left[\sum_{j \in I} \int_{\mathbb{R}} \varphi\left(a_{j}\left(a_{i}\left(r-b_{1}\right)-b_{2}\right)\right) \mu_{j}\left(d b_{2}\right)\right] \mu_{i}\left(d b_{1}\right) .
$$


Property $\mathrm{P} 1$ asserts that $a_{i}\left(r-b_{1}\right) \in D_{n+1}$ for every $i \in J$ and $b_{1} \in \operatorname{supp} \mu_{i}$. Moreover, property P2 implies that $a_{1}\left(a_{i}\left(r-b_{1}\right)-b_{2}\right) \in D_{n}$ for every $i \in J$ and $\left(a_{i}, b_{1}\right),\left(a_{1}, b_{2}\right) \in \operatorname{supp} \mu$. Since $\varphi_{\mid D_{n}}=0$, we have

$$
\varphi\left(a_{1}\left(a_{i}\left(r-b_{1}\right)-b_{2}\right)\right)=0 \text { for every } i \in J \text { and }\left(a_{i}, b_{1}\right),\left(a_{1}, b_{2}\right) \in \operatorname{supp} \mu \text {. }
$$

Hence and from the fact that $\varphi$ is bounded above by 1 we have for all $i \in I$ the inequalities

$$
\begin{aligned}
\int_{\mathbb{R}} \varphi\left(a_{i}\left(r-b_{1}\right)\right) \mu_{i}\left(d b_{1}\right) & =\int_{\mathbb{R}}\left[\sum_{j \in I} \int_{\mathbb{R}} \varphi\left(a_{j}\left(a_{i}\left(r-b_{1}\right)-b_{2}\right)\right) \mu_{j}\left(d b_{2}\right)\right] \mu_{i}\left(d b_{1}\right) \\
& =\int_{\mathbb{R}}\left[\sum_{j \in J} \int_{\mathbb{R}} \varphi\left(a_{j}\left(a_{i}\left(r-b_{1}\right)-b_{2}\right)\right) \mu_{j}\left(d b_{2}\right)\right] \mu_{i}\left(d b_{1}\right) \\
& \leq \int_{\mathbb{R}}\left[\sum_{j \in J} \int_{\mathbb{R}} \mu_{j}\left(d b_{2}\right)\right] \mu_{i}\left(d b_{1}\right) \\
& =\int_{\mathbb{R}}\left[\sum_{j \in J} \mu_{j}(\mathbb{R})\right] \mu_{i}\left(d b_{1}\right)=\mu_{i}(\mathbb{R}) \sum_{j \in J} \mu_{j}(\mathbb{R}) \\
& =\mu_{i}(\mathbb{R})\left(1-\mu_{1}(\mathbb{R})\right) .
\end{aligned}
$$

If we put the above estimations into the equality

$$
\varphi(r)=\sum_{i \in J} \int_{\mathbb{R}} \varphi\left(a_{i}(r-b)\right) \mu_{i}(d b),
$$

then we will get

$$
\varphi(r) \leq\left(1-\mu_{1}(\mathbb{R})\right) \sum_{i \in J} \mu_{i}(\mathbb{R})=\left(1-\mu_{1}(\mathbb{R})\right)^{2} .
$$

In a similar way, using P1 and P2 several times and taking into account that the function $\varphi$ vanishes on $D_{n}$ and the fact that $\varphi$ satisfies equation (2.2), one can inductively show that

$$
\varphi(r)=\sum_{i_{1}, \ldots, i_{q} \in J} \int_{\mathbb{R}^{q}} \varphi\left(a_{i_{q}}\left(\ldots\left(a_{i_{1}}\left(r-b_{1}\right)-b_{2}\right) \ldots-b_{i_{q}}\right)\right) \mu^{\otimes_{\left(i_{1}, \ldots, i_{q}\right)}}\left(d b_{1}, \ldots, d b_{q}\right),
$$

where $q \in \mathbb{N}$ and $\mu^{\otimes_{\left(i_{1}, \ldots, i_{q}\right)}}$ denotes the product of $\mu_{i_{1}}, \ldots, \mu_{i_{q}}$. Therefore, since all values of $\varphi$ lie in $[0,1]$, we have

$$
\varphi(r) \leq\left(1-\mu_{1}(\mathbb{R})\right)^{q} \text { for every } q \in \mathbb{N},
$$

and thus $\varphi(r)=0$ because of the condition $\mu_{1}(\mathbb{R}) \in(0,1)$. Consequently, we get $\varphi(x)=0$ for every $x \in D_{n+1}$. Summarizing we see that $\varphi$ vanishes on $D$. 
Theorem 2.4 may be reformulated also in the language of random variables because of the equivalence between equations (1.1) and (1.2).

Theorem 2.6. If $\mathbb{P}(\alpha \in(-\infty, 0) \backslash\{-1\})>0$ and there exists $b \in \mathbb{R} \backslash\{0\}$ such that $\mathbb{P}((\alpha, \beta) \in U)>0$ for each open neighborhood $U$ of $(1, b)$, then every bounded continuous solution of equation (1.2) attaining its global extremum is constant.

Remark 2.7. The assertation of Theorem 2.4 is false when $\mu(\{-1,1\} \times \mathbb{R})=1$. This follows from [4, Theorem 2.3].

The condition $(1, b) \in \operatorname{supp} \mu$, where $b \neq 0$, is not satisfied for all measures. Thus, we are going to prove the next theorem giving another condition under which every bounded continuous solution of the archetypal equation attaining the global extremum is constant. We start with a very simple fact.

Lemma 2.8. Let $a \in(-1,1) \backslash\{0\}$ and $t \in(0,+\infty)$. If $\left(u_{n}\right)_{n \in \mathbb{N} \cup\{0\}}$ is a sequence of negative numbers, then the set

$$
\bigcup_{n=1}^{+\infty}\left\{u_{n}+a^{n} k t: k \in \mathbb{N} \cup\{0\}\right\}
$$

is dense in the positive half line.

Proof. Let $x, \varepsilon \in(0,+\infty)$ be fixed. Choose $n_{0} \in \mathbb{N}$ such that $a^{2 n_{0}} t<\varepsilon$ and take any $n \geq n_{0}$. Note that $a^{2 n}>0$, thus

$$
(0,+\infty) \subset \bigcup_{k=0}^{+\infty}\left[u_{2 n}+a^{2 n} k t, u_{2 n}+a^{2 n}(k+1) t\right) .
$$

We can find $k_{0} \in \mathbb{N} \cup\{0\}$ such that $x \in\left[u_{2 n}+a^{2 n} k_{0} t, u_{2 n}+a^{2 n}\left(k_{0}+1\right) t\right)$. In the end, observe that the length of this interval is less than $\varepsilon$.

Theorem 2.9. If $\mu((-\infty, 0) \times \mathbb{R})>0$ and there exist $\left(a_{1}, b_{1}\right), \ldots,\left(a_{s}, b_{s}\right)$ from supp $\mu$ such that $\min \left\{\left|a_{1}\right|, \ldots,\left|a_{s}\right|\right\}<1, a_{1} \cdot \ldots \cdot a_{s}=-1$ and

$$
\sum_{i=1}^{2 s} a_{\sigma(2 s)} \cdot \ldots \cdot a_{\sigma(i)} b_{\sigma(i)} \neq 0,
$$

for some integer $s \geq 2$ and $\sigma:\{1,2, \ldots, 2 s\} \rightarrow\{1,2, \ldots, s\}$ which takes each value exactly twice, then every continuous solution $\varphi: \mathbb{R} \rightarrow \mathbb{R}$ of equation (1.1) attaining the global extremum is constant.

Proof. We may assume without loss of generality that inf $\varphi(\mathbb{R})=0$ and there exists $x_{0} \in \mathbb{R}$ such that $\varphi\left(x_{0}\right)=0$. Observe that the assumptions imposed on $a_{1}, \ldots, a_{s}$ and $\sigma$ give

$$
a_{\sigma(1)} \cdot \ldots \cdot a_{\sigma(2 s)}=\left(a_{1} \cdot \ldots \cdot a_{s}\right)^{2}=(-1)^{2}=1 .
$$


If we apply Lemma 2.2 with $\left(c_{i}, d_{i}\right)=\left(a_{\sigma(i)}, b_{\sigma(i)}\right)$ for $i=1, \ldots, 2 s$ to the point $x_{0}$, we will get

$$
\begin{gathered}
\varphi\left(a_{\sigma(1)} \cdot \ldots \cdot a_{\sigma(2 s)} x_{0}-\sum_{i=1}^{2 s} a_{\sigma(2 s)} \cdot \ldots \cdot a_{\sigma(i)} b_{\sigma(i)}\right) \\
=\varphi\left(x_{0}-\sum_{i=1}^{2 s} a_{\sigma(2 s)} \cdot \ldots \cdot a_{\sigma(i)} b_{\sigma(i)}\right)=0 .
\end{gathered}
$$

Denote $-\sum_{i=1}^{2 s} a_{\sigma(2 s)} \cdot \ldots . a_{\sigma(i)} b_{\sigma(i)}$ by $t$ for simplicity. Then the last equality can be rewritten as

$$
\varphi\left(x_{0}+t\right)=0 \text {. }
$$

If we again use Lemma 2.2 with $\left(c_{i}, d_{i}\right)=\left(a_{\sigma(i)}, b_{\sigma(i)}\right)$ for $i=1, \ldots, 2 s$ to the point $x_{0}+t$, then we will get $\varphi\left(x_{0}+2 t\right)=0$. By the induction one can prove that

$$
\varphi\left(x_{0}+k t\right)=0 \text { for every } k \in \mathbb{N} \cup\{0\} .
$$

Now fix any $k \in \mathbb{N} \cup\{0\}$. Using Lemma 2.2 with $\left(c_{i}, d_{i}\right)=\left(a_{i}, b_{i}\right)$ for every $i=1,2, \ldots, s$ to the point $x_{0}+k t$, we come to

$$
\varphi\left(a_{s} \cdot \ldots \cdot a_{1}\left(x_{0}+k t\right)-\sum_{i=1}^{s} a_{s} \cdot \ldots \cdot a_{i} b_{i}\right)=\varphi\left(-x_{0}-k t-\sum_{i=1}^{s} a_{s} \cdot \ldots \cdot a_{i} b_{i}\right)=0
$$

since $a_{1} \cdot \ldots \cdot a_{s}=-1$. Therefore we have

$$
\varphi\left(y_{0}-k t\right)=0 \text { for all } k \in \mathbb{N} \cup\{0\},
$$

where $y_{0}:=-x_{0}-\sum_{i=1}^{s} a_{s} \ldots a_{i} b_{i}$.

Without loss of generality we may assume that $\left|a_{1}\right|<1$. Let $k \in \mathbb{N} \cup\{0\}$ and $n \in \mathbb{N}$ be taken arbitrarily. If we use Lemma 2.2 with $\left(c_{i}, d_{i}\right)=\left(a_{1}, b_{1}\right)$ for every $i=1,2, \ldots, n$ to the points $x_{0}+k t$ and $y_{0}-k t$, then we obtain the equalities

$$
\varphi\left(a_{1}^{n} x_{0}-\sum_{j=1}^{n} a_{1}^{j} b_{1}+a_{1}^{n} k t\right)=0
$$

and

$$
\varphi\left(a_{1}^{n} y_{0}-\sum_{j=1}^{n} a_{1}^{j} b_{1}-a_{1}^{n} k t\right)=0
$$

respectively.

We know that $t \neq 0$, that is either positive, or negative. We consider the first case. Conditions (2.7) and (2.8) imply that the set of zeros of the function $\varphi$ is unbounded both from above and from below. Therefore for every $n \in \mathbb{N}$ we can choose a zero $u_{n} \in \mathbb{R}$ of the function $\varphi$ such that

$$
a_{1}^{n} u_{n}-\sum_{j=1}^{n} a_{1}^{j} b_{1}<0 .
$$


We define also a sequence $\left(v_{n}\right)_{n \in \mathbb{N}}$ of zeros of the function $\varphi$ fulfilling the opposite inequalities

$$
a_{1}^{n}\left(-v_{n}-\sum_{i=1}^{s} a_{s} \ldots a_{i} b_{i}\right)-\sum_{j=1}^{n} a_{1}^{j} b_{1}>0 \text { for every } n \in \mathbb{N} .
$$

Equalities (2.9) and (2.10) imply that for every $n \in \mathbb{N}$ and $k \in \mathbb{N} \cup\{0\}$ we have

$$
\varphi\left(a_{1}^{n} u_{n}-\sum_{j=1}^{n} a_{1}^{j} b_{1}+a_{1}^{n} k t\right)=0
$$

and

$$
\varphi\left(a_{1}^{n}\left(-v_{n}-\sum_{i=1}^{s} a_{s} \ldots a_{i} b_{i}\right)-\sum_{j=1}^{n} a_{1}^{j} b_{1}-a_{1}^{n} k t\right)=0 .
$$

Put

$$
E:=\bigcup_{n=1}^{+\infty}\left\{a_{1}^{n} u_{n}-\sum_{j=1}^{n} a_{1}^{j} b_{1}+a_{1}^{n} k t: k \in \mathbb{N} \cup\{0\}\right\}
$$

and

$$
F:=\bigcup_{n=1}^{+\infty}\left\{a_{1}^{n}\left(-v_{n}-\sum_{i=1}^{s} a_{s} \ldots a_{i} b_{i}\right)-\sum_{j=1}^{n} a_{1}^{j} b_{1}-a_{1}^{n} k t: k \in \mathbb{N} \cup\{0\}\right\} .
$$

Then $\varphi_{\mid E \cup F}=0$. Lemma 2.8 asserts that $E$ and $-F$ are dense in $(0,+\infty)$. Hence $E \cup F$ is dense in $\mathbb{R}$, and thus $\varphi$ is constant. If $t$ is negative, the proof is similar and we omit it.

If $s=2$ condition (2.6) reduces to a simpler form which is easy to check.

Corollary 2.10. Assume that $\mu((-\infty, 0) \times \mathbb{R})>0$ and there exist $\left(a_{1}, b_{1}\right),\left(a_{2}, b_{2}\right)$ from supp $\mu$ such that $\left|a_{1}\right| \neq 1, a_{1} a_{2}=-1$ and

$$
b_{2} \neq \frac{a_{1}^{2}+a_{1}}{a_{1}-1} b_{1} .
$$

Then every continuous solution $\varphi: \mathbb{R} \rightarrow \mathbb{R}$ of equation (1.1) attaining the global extremum is constant.

Proof. We shall check that all assumptions of Theorem 2.9 are satisfied. Obviously conditions $\left|a_{1}\right| \neq 1$ and $a_{1} a_{2}=-1$ imply that either $\left|a_{1}\right|<1$ or $\left|a_{2}\right|<1$. It remains to check (2.6). We can treat $\sigma$ as a sequence $(\sigma(1), \sigma(2), \sigma(3), \sigma(4))$ 
of digits 1 and 2, each of which appears twice. We will see that condition (2.6) holds if $\sigma=(1,1,2,2)$. Putting equality $a_{2}=-\frac{1}{a_{1}}$ into the sum

$$
\sum_{i=1}^{2 s} a_{\sigma(2 s)} \cdot \ldots \cdot a_{\sigma(i)} b_{\sigma(i)}
$$

we get

$$
a_{1}^{2} a_{2}^{2} b_{1}+a_{1} a_{2}^{2} b_{1}+a_{2}^{2} b_{2}+a_{2} b_{2}=b_{1}+\frac{1}{a_{1}} b_{1}+\frac{1}{a_{1}^{2}} b_{2}-\frac{1}{a_{1}} b_{2} .
$$

The above expression takes value 0 if and only if

$$
\left(1+\frac{1}{a_{1}}\right) b_{1}=\left(\frac{1}{a_{1}}-\frac{1}{a_{1}^{2}}\right) b_{2},
$$

that is

$$
b_{2}=\frac{a_{1}^{2}+a_{1}}{a_{1}-1} b_{1}
$$

Since we assumed that $b_{2} \neq \frac{a_{1}^{2}+a_{1}}{a_{1}-1} b_{1}$, condition (2.6) is satisfied.

Unfortunately the class of measures from Theorems 2.4 and 2.9 still do not cover all possibilities. Consider the following

Example. Let $\mu$ be a probability measure on the plane $\mathbb{R}^{2}$ such that $\operatorname{supp} \mu=$ $\{(-2,1),(2,2)\}$. Then we cannot use Theorem 2.4 since there is no point of the form $(1, b)$ with some $b \neq 0$ in $\operatorname{supp} \mu$. We cannot use Theorem 2.9 either as $\operatorname{supp} \mu$ does not contain a point of the form $(a, b) \in \mathbb{R}^{2}$ with $|a|<1$.

\section{The asymptotics of solutions}

In this part we study the asymptotic behaviour of bounded continuous solutions of (1.1) which do not necessarily attain the global extremes.

Theorem 3.1. Assume that $\mu(((-\infty, 0) \backslash\{-1\}) \times \mathbb{R})>0$ and there exists $b \in$ $\mathbb{R} \backslash\{0\}$ such that $(1, b) \in \operatorname{supp} \mu$. Let $\varphi: \mathbb{R} \rightarrow \mathbb{R}$ be a bounded continuous solution of (1.1). Then

$$
\liminf _{x \rightarrow-\infty} \varphi(x)=\liminf _{x \rightarrow+\infty} \varphi(x)=\inf \varphi(\mathbb{R})
$$

and

$$
\limsup _{x \rightarrow-\infty} \varphi(x)=\limsup _{x \rightarrow+\infty} \varphi(x)=\sup \varphi(\mathbb{R}) .
$$


Proof. We will show equalities (3.1) only. Choose a sequence $\left(x_{n}\right)_{n \in \mathbb{N}}$ of reals such that

$$
\varphi\left(x_{n}\right) \rightarrow \inf \varphi(\mathbb{R}) .
$$

If it is bounded, then one can find its subsequence $\left(y_{n}\right)_{n \in \mathbb{N}}$ which is convergent to some $x_{0} \in \mathbb{R}$. Then, by the continuity of $\varphi$, we have $\varphi\left(x_{0}\right)=\inf \varphi(\mathbb{R})$. Hence, by Theorem 2.4, we know that $\varphi$ is constant and equalities (3.1) hold. So we may assume that $\left(x_{n}\right)_{n \in \mathbb{N}}$ is unbounded. Then we can choose a subsequence $\left(y_{n}\right)_{n \in \mathbb{N}}$ such that either $y_{n} \rightarrow-\infty$, or $y_{n} \rightarrow+\infty$. Consider, for instance, the first possibility. Then

$$
\lim _{n \rightarrow+\infty} \varphi\left(y_{n}\right)=\inf \varphi(\mathbb{R}),
$$

and thus

$$
\liminf _{x \rightarrow-\infty} \varphi(x)=\inf \varphi(\mathbb{R}) .
$$

Since, in view of Theorem 4.2 from [3], we have

$$
\liminf _{x \rightarrow-\infty} \varphi(x)=\liminf _{x \rightarrow+\infty} \varphi(x),
$$

we come to (3.1).

From this theorem the following result can be immediately deduced.

Corollary 3.2. Assume that $\mu(((-\infty, 0) \backslash\{-1\}) \times \mathbb{R})>0$ and there exists $b \in$ $\mathbb{R} \backslash\{0\}$ such that $(1, b) \in$ supp $\mu$. Let $\varphi: \mathbb{R} \rightarrow \mathbb{R}$ be a bounded continuous solution of (1.1). If at least one of the limits $\lim _{x \rightarrow-\infty} \varphi(x)$ and $\lim _{x \rightarrow+\infty} \varphi(x)$ exists, then $\varphi$ is constant.

We also have the analogous results generated by Theorem 2.9.

Theorem 3.3. Assume that $\mu((-\infty, 0) \times \mathbb{R})>0$ and the set supp $\mu$ contains $\left(a_{1}, b_{1}\right), \ldots,\left(a_{s}, b_{s}\right)$ such that $\min \left\{\left|a_{1}\right|, \ldots,\left|a_{s}\right|\right\}<1, a_{1} \cdot \ldots \cdot a_{s}=-1$ and condition (2.6) is satisfied for some integer $s \geq 2$ and $\sigma:\{1,2, \ldots, 2 s\} \rightarrow$ $\{1,2, \ldots, s\}$ which takes value exactly twice. Let $\varphi: \mathbb{R} \rightarrow \mathbb{R}$ be a bounded continuous solution of (1.1). Then

$$
\liminf _{x \rightarrow-\infty} \varphi(x)=\liminf _{x \rightarrow+\infty} \varphi(x)=\inf \varphi(\mathbb{R})
$$

and

$$
\limsup _{x \rightarrow-\infty} \varphi(x)=\limsup _{x \rightarrow+\infty} \varphi(x)=\sup \varphi(\mathbb{R}) .
$$

Corollary 3.4. Assume that $\mu((-\infty, 0) \times \mathbb{R})>0$ and the set supp $\mu$ contains $\left(a_{1}, b_{1}\right), \ldots,\left(a_{s}, b_{s}\right)$ such that $\min \left\{\left|a_{1}\right|, \ldots,\left|a_{s}\right|\right\}<1, a_{1} \cdot \ldots \cdot a_{s}=-1$ and condition (2.6) is satisfied for some integer $s \geq 2$ and $\sigma:\{1,2, \ldots, 2 s\} \rightarrow$ $\{1,2, \ldots, s\}$ which takes value exactly twice. Let $\varphi: \mathbb{R} \rightarrow \mathbb{R}$ be a bounded continuous solution of (1.1). If at least one of the limits $\lim _{x \rightarrow-\infty} \varphi(x)$ and $\lim _{x \rightarrow+\infty} \varphi(x)$ exists, then $\varphi$ is constant. 
The above corollaries can be compared with a similar theorem by Bogachev, Derfel and Molchanov (see [3, Theorem 4.3]).

Theorem BDM Assume that the measure $\mu$ fulfills the conditions: $\mu((-\infty, 0) \times$ $\mathbb{R})>0, \mu(\{0\} \times \mathbb{R})=0, \mu\left(\left\{(a, b) \in \mathbb{R}^{2}: a(c-b)=c\right\}\right)<1$ for all $c \in \mathbb{R}$ and $\mu(\{-1,1\} \times \mathbb{R})<1$. Assume that $K \in(0,+\infty)$ and $\iint_{\mathbb{R}^{2}} \ln (\max (|b|, 1)) \mu(d a, d b)<$ $+\infty$. Let $\varphi: \mathbb{R} \rightarrow \mathbb{R}$ be a bounded solution of equation (1.1). If at least one of the limits $\lim _{x \rightarrow-\infty} \varphi(x)$ and $\lim _{x \rightarrow+\infty} \varphi(x)$ exists, then $\varphi$ is constant.

The below examples show that the above results are not comparable: none of them implies the other.

Example. Assume that $\mu(\{(1,1)\})=1 / 2$ and

$$
\mu\left(\left\{\left(-\mathrm{e}^{2^{n}}, 0\right)\right\}\right)=\frac{1}{2^{n}} \text { for } n=2,3, \ldots
$$

Note that

$$
K=\frac{1}{2} \ln |1|+\sum_{n=2}^{+\infty} \frac{1}{2^{n}} \ln \left|-\mathrm{e}^{2^{n}}\right|=\sum_{n=2}^{+\infty} 1=+\infty .
$$

Moreover $\mu(((-\infty,-1) \cup(-1,0)) \times \mathbb{R})=1 / 2$. In this case Theorem BDM cannot be used since $K=+\infty$ but Corollary 3.2 can be applied because of the inequalities $\mu(((-\infty,-1) \cup(-1,0)) \times \mathbb{R})>0$ and $\mu(\{(1,1)\})>0$; the second inequality is sufficient since the measure $\mu$ is purely atomic. Thus each bounded continuous solution $\varphi: \mathbb{R} \rightarrow \mathbb{R}$ of the equation

$$
\varphi(x)=\frac{1}{2} \varphi(x-1)+\sum_{n=2}^{+\infty} \frac{1}{2^{n}} \varphi\left(-\mathrm{e}^{2^{n}} x\right)
$$

having at least one of the limits $\lim _{x \rightarrow-\infty} \varphi(x)$ and $\lim _{x \rightarrow+\infty} \varphi(x)$ is constant.

If we change the above example only in one place, we can apply neither Corollaries 3.2 and 3.4, nor Theorem BDM.

Example. Assume that $\mu(\{(\mathrm{e}, 1)\})=1 / 2$ and

$$
\mu\left(\left\{\left(-\mathrm{e}^{2^{n}}, 0\right)\right\}\right)=\frac{1}{2^{n}} \text { for } n=2,3, \ldots
$$

For such a measure $\mu$ we cannot use Corollary 3.2 since there is no (for a measure defined like that) $b \in \mathbb{R} \backslash\{0\}$ such that $\mu(\{(1, b)\})>0$. Further, observe that if $\mu(\{(a, b)\})>0$, then $|a|>1$. Therefore we cannot apply Corollary 3.4 in this example. Theorem BDM cannot be used here either for the same reason as in the previous example. We know nothing about nonconstant bounded continuous solutions $\varphi: \mathbb{R} \rightarrow \mathbb{R}$ of the equation

$$
\varphi(x)=\frac{1}{2} \varphi(\mathrm{e} x-\mathrm{e})+\sum_{n=2}^{+\infty} \frac{1}{2^{n}} \varphi\left(-\mathrm{e}^{2^{n}} x\right) .
$$


In the last example Theorem BDM can be applied but Corollaries 3.2 and 3.4 cannot.

Example. Assume that $\mu(\{(-1,1)\})=\mu(\{(-\mathrm{e}, 0)\})=1 / 2$. All assumptions from Theorem BDM are satisfied. Indeed

$$
K=\frac{1}{2} \ln |-1|+\frac{1}{2} \ln |-\mathrm{e}|=\frac{1}{2}<+\infty
$$

and

$$
\begin{aligned}
\iint_{\mathbb{R}^{2}} \ln (\max (|b|, 1)) & \mu(d a, d b) \\
& =\frac{1}{2} \ln (\max (|1|, 1))+\frac{1}{2} \ln (\max (|\mathrm{e}|, 1))=\frac{1}{2}<+\infty .
\end{aligned}
$$

Corollaries 3.2 and 3.4 cannot be used here for the same reasons as in the previous example. Therefore, in view of Theorem BDM, every bounded continuous solution $\varphi: \mathbb{R} \rightarrow \mathbb{R}$ of the equation

$$
\varphi(x)=\frac{1}{2} \varphi(-x-1)+\frac{1}{2} \varphi(-\mathrm{e} x)
$$

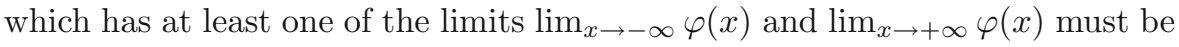
constant.

In conclusion, the presented theorems show that non-constant solutions of the archetypal equation in the case $\mu((-\infty, 0) \times \mathbb{R})>0$ have to be oscillating functions. The question of the existence of bounded continuous solutions, which are not constant, is still an open problem.

Open Access. This article is licensed under a Creative Commons Attribution 4.0 International License, which permits use, sharing, adaptation, distribution and reproduction in any medium or format, as long as you give appropriate credit to the original author(s) and the source, provide a link to the Creative Commons licence, and indicate if changes were made. The images or other third party material in this article are included in the article's Creative Commons licence, unless indicated otherwise in a credit line to the material. If material is not included in the article's Creative Commons licence and your intended use is not permitted by statutory regulation or exceeds the permitted use, you will need to obtain permission directly from the copyright holder. To view a copy of this licence, visit http:// creativecommons.org/licenses/by/4.0/.

Publisher's Note Springer Nature remains neutral with regard to jurisdictional claims in published maps and institutional affiliations.

\section{References}

[1] Baron, K., Jarczyk, W.: Recent results on functional equations in a single variable, perspectives and open problems. Aequ. Math. 61, 1-48 (2001)

[2] Baron, K., Morawiec, J.: Lipschitzian solutions to linear iterative equations revisited. Aeq. Math. 91, 161-167 (2017) 
[3] Bogachev, L.V., Derfel, G., Molchanov, S.A.: Analysis of the archetypal functional equation in the non-critical case. In: AIMS Proceedings, Springfield, pp. 132-141 (2015)

[4] Bogachev, L.V., Derfel, G., Molchanov, S.A.: On bounded continuous solutions of the archetypal equation with rescaling. Proc. R. Soc. A471, 1-19 (2015)

[5] Derfel, G.: Probabilistic method for a class of functional-differential equations. Ukr. Math. J. 41, 1137-1141 (1989)

[6] Derfel, G.: Functional-differential and functional equations with rescaling. In: Operator Theory and Boundary Eigenvalue Problems, vol. 80 (eds Gohberg I, Langer H), Operator Theory: Advances and Applications, Basel, Switzerland: Birkhäuser, pp. 100-111 (1995)

[7] Hardy, G.H., Wright, E.M.: An Introduction to the Theory of Numbers. Oxford University Press, London (1960)

[8] Kapica, R., Morawiec, J.: Continuous solutions of iterative equations of infinite order. Opusc. Math. 29, 147-155 (2009)

[9] Sudzik, M.: On a functional equation related to a problem of G. Derfel. Aequat. Math. 93, 137-148 (2019)

\section{Mariusz Sudzik}

Institute of Mathematics

University of Zielona Góra

Szafrana $4 \mathrm{a}$

65-516 Zielona Góra

Poland

e-mail: M.Sudzik@wmie.uz.zgora.pl

Received: April 12, 2020

Revised: October 31, 2020

Accepted: November 3, 2020 